\title{
Crystallization of silicon nanoclusters with inert gas temperature control
}

\author{
Junlei Zhao, ${ }^{1}$ Vidyadhar Singh, ${ }^{2}$ Panagiotis Grammatikopoulos, ${ }^{2}$ Cathal Cassidy, ${ }^{2}$ Kengo Aranishi, ${ }^{2}$ Mukhles Sowwan, 2,3 \\ Kai Nordlund, ${ }^{1}$ and Flyura Djurabekova ${ }^{1}$ \\ ${ }^{1}$ Department of Physics and Helsinki Institute of Physics, University of Helsinki, P.O. Box 43, FIN-00014 Helsinki, Finland \\ ${ }^{2}$ Nanoparticles by Design Unit, Okinawa Institute of Science and Technology (OIST) Graduate University, \\ 1919-1 Onna-Son, Okinawa, 904-0495, Japan \\ ${ }^{3}$ Nanotechnology Research Laboratory, Al-Quds University, East Jerusalem, P.O. Box 51000, Palestine \\ (Received 22 September 2014; revised manuscript received 9 December 2014; published 15 January 2015)
}

\begin{abstract}
We analyze the fundamental process of crystallization of silicon nanoclusters by means of molecular dynamics simulations, complemented by magnetron-sputter inert gas condensation, which was used to synthesize polycrystalline silicon nanoclusters with good size control. We utilize two well-established Si interatomic potentials: the Stillinger-Weber and the Tersoff III. Both the simulations and experiments show that upon cooling down by an Ar gas thermal bath, initially liquid, free-standing Si nanocluster can grow multiple crystal nuclei, which drive their transition into polycrystalline solid nanoclusters. The simulations allow detailed analysis of the mechanism, and show that the crystallization temperature is size-dependent and that the probability of crystalline phase nucleation depends on the highest temperature the cluster reaches during the initial condensation and the cooling rate after it.
\end{abstract}

DOI: 10.1103/PhysRevB.91.035419

PACS number(s): 81.07.Bc, 81.10.St

\section{INTRODUCTION}

One of the key challenges in nanotechnology today is to control the size and crystallinity of silicon nanoclusters ( $\mathrm{Si}$ NCs) with a high degree of accuracy, as these parameters have an important impact on uses in specific biomedical and optoelectronic applications [1-3]. Conventional thin-film particle synthesis systems suffer from insufficient simultaneous control of both particle size and crystallinity, especially in the smaller diameter regime. The inert gas condensation technique, however, can overcome this limitation, providing good control over both of these properties.

Research on Si NCs has been very active over the years, given the material's prevalent position in modern technology. For instance, crystallization of Si NCs from gas phase condensation is particularly interesting for the $\mathrm{Si}$-based photovoltaic industry [4-6]. In general, even though it is of course very well known that the same material can exist in liquid, ordered (crystalline) and disordered (amorphous) solid phases depending on temperature and rate of cooling [7], the fundamental atom level mechanisms behind the phase transitions between these are not fully established and are subject to recent research interest [8-14]. Nanosystems provide an interesting addition to these studies as in them, the surface free energy is a significant contribution to the whole, and thus can provide additional insight into the fundamental thermodynamics of melting.

To elucidate melting and crystallization mechanisms, the existing experimental tools may not be sufficient, as they cannot track atom motion in a multiphase system at the intrinsic lattice vibration rates around $\sim 10^{13} \mathrm{~Hz}$. Computer simulation methods, which describe the atomic interactions, can be employed instead, since the time and spatial scales

Published by the American Physical Society under the terms of the Creative Commons Attribution 3.0 License. Further distribution of this work must maintain attribution to the author(s) and the published article's title, journal citation, and DOI. involved in the phase transition processes are well within their resolution capabilities. Yet computer simulation results alone cannot give a conclusive understanding of the physics of studied processes, unless the simulation results are validated against experimental observations. Hence, in the current work, we combine simulations and experiments of Si crystal condensation to elucidate the mechanisms of cluster formation, with a particular emphasis on examining the conditions under which the clusters can crystallize.

Recrystallization of bulk Si has been successfully studied by using molecular dynamics simulation methods $[15,16]$. For instance, it was previously shown that crystallization occurs just below the high-density to low-density liquid transition temperature: the Stillinger-Weber potential, which describes well that the low-density liquid phase of $\mathrm{Si}$ is thermodynamically and structurally contiguous to the amorphous solid, allows for the rapid crystallization at $1050 \mathrm{~K}[17,18]$. A high-density liquid phase did not result in recrystallization, as shown in Ref. [18]. Later on, the same authors did observe recrystallization of bulk $\mathrm{Si}$ without going through the lowdensity liquid phase at $0.75 T_{\text {melting }}$ [19].

The properties of materials at the nanoscale are frequently defined by a high fraction of surface atoms, which makes the behavior of nanosystem different from that of the corresponding bulk material. For the study of such nanoscale processes, a special attention must be paid to how the simulations are conducted. For example, special thermo- and barostats are required to control the simulation conditions compared to the conventional ones used to study the bulk materials. The use of the Nosé-Hoover thermostat [20,21] or its extension, the Anderson thermostat [22], can cause significant artifacts in the thermodynamic process of an NC system since these methods do not distinguish between surface and bulk atoms. Such artificial scaling of velocities in the nanocluster can be avoided by applying the inert gas temperature control [23], which moreover allows mimicking of the experimental conditions in a nanoparticle deposition chamber [24].

The interatomic potential in use must also meet certain requirements for realistic description of interatomic interactions. 
The condensation process of Si NCs can be describe properly, if the potential: (i) describes all three phases fairly well, (ii) yields the phase transition temperatures close to the experimental values, and (iii) reproduces the bond angle energy and bond angle distribution for solid Si correctly. The latter is particularly important for crystallization transition stage. Unfortunately, none of the empirical potentials developed to date can fully reproduce all three phases of silicon: crystalline, amorphous, and liquid [25-27].

In this paper, we study the crystallization process of a molten Si NC, by combination of simulation and experimental methods. We investigate the possible mechanisms that lead to crystallization by molecular dynamics (MD) simulation methods and propose that the simultaneous growth of multiple seeds explain the final polycrystalline structure of deposited NCs. To validate the simulation results and their interpretation, we compare them to the experimental results of polycrystalline Si NCs grown by magnetron sputter inert gas condensation method.

\section{METHODS}

\section{A. Simulation}

In this work, the MD simulations were set up so that they can be categorized in three groups: (i) 4-nm cluster case: 1677 $\mathrm{Si}$ atoms, $1350 \mathrm{Ar}$ atoms in $40 \mathrm{~nm} \times 40 \mathrm{~nm} \times 40 \mathrm{~nm}$ box; (ii) 9-nm cluster case: $20475 \mathrm{Si}$ atoms, 1194/900 Ar atoms in $11 \mathrm{~nm} \times 11 \mathrm{~nm} \times 11 \mathrm{~nm}$ box; and (iii) $4-\mathrm{nm}$ cluster case with various number of Ar atoms: $1677 \mathrm{Si}$ atoms, 200/150/100/50 $\mathrm{Ar}$ atoms in $6 \mathrm{~nm} \times 6 \mathrm{~nm} \times 6 \mathrm{~nm}$ box. The pure Si NC in the liquid phase was initially placed in the center of the simulation cell. The surrounding atmosphere consisted of isolated $\mathrm{Ar}$ atoms, randomly distributed around the $\mathrm{Si}$ $\mathrm{NC}$, as shown in Fig. 1. The initial velocities of all atoms were generated in a Maxwell-Boltzmann distribution [28] to ensure a desired initial temperature in all simulations. For each specific combination of Si and Ar atoms, the initial temperature of $\mathrm{Si}$ atoms was selected to ensure that the initial state of the $\mathrm{Si}$ $\mathrm{NC}$ was in a hot liquid phase, but no evaporation yet occurred. In our simulations, we omitted the condensation process of Si NC for two reasons: (i) the previous studies [23,29-35] as well as our analytical estimation (see Fig. 14 and Appendix A) indicate that the NCs condensed in the plasma region, where the temperature is a few thousands degrees of Kelvin, are initially in the liquid (molten) phase (we refer to these nanoclusters, hereafter, as "liquid" NCs); (ii) it is extremely slow to simulate sufficiently large NCs from the gas phase.
The initial liquid structure of the 4-nm cluster was obtained by heating the cluster to $2300 \mathrm{~K}$. The 9 -nm cluster was cut from an amorphous Si thin film generated by the Wooten, Winer, and Weaire (WWW) method [36]. The two different methods of generating disordered silicon phase have been used in previous studies [37-39]. We note that melting a 9-nm Si NC without any evaporation is quite difficult, so we used instead the WWW method. Using two different procedures to obtain molten silicon nanoclusters also allows to avoid a possible bias caused by a specific method.

Simulations were done by using the PARCAS code [40,41]. To obtain the interatomic potential independent results, two common and well-established Si potentials [25] were used, the Stillinger-Weber (SW) [42], and Tersoff III (T-III) [43]. The T-III potential has the shortest range of interactions (2.85 $\AA$ ) amongst existing silicon potentials. The parameters of the Tersoff potentials were fitted to an $a b$ initio database of different structures of silicon, but not to any data corresponding to the liquid phase. Also, the T-III potential was found to overestimate the melting point of silicon significantly [40]. On the other hand, it describes various silicon crystal structures fairly well.

The cutoff radius of the SW potential is $3.771 \AA$. The SW potential describes crystalline, amorphous and liquid phases fairly well. The difference between the two potentials may affect dynamic properties of Si during the liquid-amorphous phase transition and amorphous-crystalline phase transition. Interactions between $\mathrm{Si}$ and $\mathrm{Ar}$ atoms were described by the purely repulsive potential developed by Ziegler, Biersack, and Littmark (ZBL) [44]. The interaction between Ar atoms was simulated by the Lennard-Jones potential [45]. We also note that the time step in the simulations with SW potential was $1.079 \mathrm{fs}$, while in the simulations with T-III potential it was set to $0.270 \mathrm{fs}$. These were chosen, based on testing in model NVE ensemble simulations, to guarantee the energy conservation in pure Si.

The temperature of Ar atoms was constantly scaled to $300 \mathrm{~K}$ by the Berendsen thermostat, whence the temperature of the whole system was reduced via the thermal bath of the Ar atmosphere. The whole system can be regarded as a canonical (NVT) ensemble but far away from the equilibrium state. The reason for using an inert gas temperature control method, instead of applying a thermostat such Berendsen or Nosé-Hoover, is that the thermodynamic process is essential in the present simulations. In the real physical process, particles are cooled via collisions with atmosphere

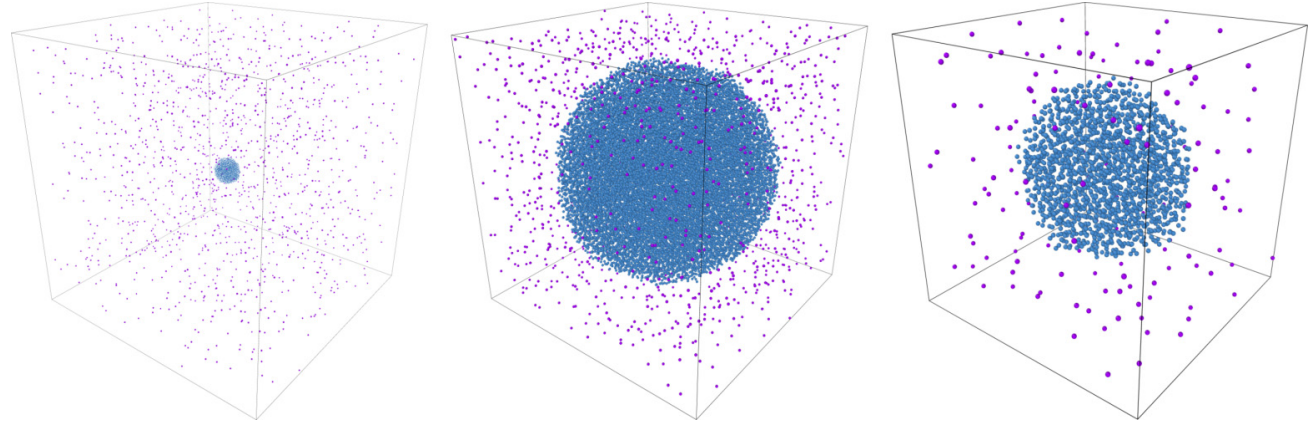

FIG. 1. (Color online) Simulation setup for 4-nm cluster case in 40-nm box (left), 9-nm cluster case in 11-nm box (middle), and 4-nm cluster in 6-nm box (right). The Si NC (blue atom) at the center surrounded by Ar atoms (purple atom). 
atoms and black-body radiation from the nanoparticle's surface. However, the latter process is significant mainly on microsecond timescales [46] and hence in the current simulations, which were run on shorter timescales, it was sufficient to consider the atomic collision cooling.

A conventional thermostat would homogeneously cool down the cluster, affecting the Maxwell-Boltzmann distribution of thermal velocities and hence, affecting the thermodynamics of the cooling process, which is important for the stochastic nature of seed formation for crystallisation process. The cooling rate of the thermal bath of Ar gas fluctuated around $10 \mathrm{~K} / \mathrm{ns}$ in the 4-nm case. Two cooling rates were used for 9-nm nanocluster, which are 20 and $30 \mathrm{~K} / \mathrm{ns}$ (see Appendix A, for the details on cooling rate estimations).

\section{Simulation analysis methods}

To quantify the degree of amorphization in a nanosystem, an angular structure factor $P_{\mathrm{st}}$ [47] is calculated for each atom $i$ :

$$
\begin{gathered}
P_{\mathrm{st}}(i)=\frac{1}{p_{u}(i)}\left[\sum_{j}\left(\theta_{i}(j)-\theta_{i}^{p}(j)\right)^{2}\right]^{1 / 2}, \\
p_{u}(i)=\left[\sum_{j}\left(\theta_{i}^{u}(j)-\theta_{i}^{p}(j)\right)^{2}\right]^{1 / 2},
\end{gathered}
$$

where $\theta_{i}(j)$ is a list of the angles formed between atom $i$ and its neighbours $j$. The number of the neighbours is determined from the ideal crystal structure, which for the diamond structure $(\mathrm{Si})$ is four. $\theta_{i}^{p}(j)$ is the distribution of angles in a perfect lattice and $\theta_{i}^{u}(j)=j \pi /\left[n_{\mathrm{nb}}\left(n_{\mathrm{nb}}-1\right) / 2\right]$ is the uniform angular distribution. $n_{\mathrm{nb}}$ is the number of the first nearest neighbors determined from the ideal crystal structure. Before doing the sum over the angles, the lists of $\theta_{i}(j)$ are sorted by magnitude. By averaging the structure factor of each atom in combination with a kinetic energy criterion, we can analyze the degree of amorphization as a function of simulation time. For the structural factor analysis, we used the cutoff radius $3.1 \AA$, which is the average value of the first and second peaks in the pair distribution function for a regular silicon crystal structure (also see Fig. 4).

We applied the ring analysis algorithm [48] to detect the primitive silicon sixfold rings in wurtzite and diamond basic block. These were used to localize the seeds for the crystalline phase. For this analysis, we reduced somewhat the cutoff radius from 3.1 to $2.39 \AA$, a distance which is slightly larger than the equilibrium interatomic distance in Si structure. This choice allowed to define the transition point clearly, excluding the nonprimitive rings which unavoidably appeared with larger cutoff radii. However, we note that varying the cutoff radius did not affect the conclusion on the time when the phase transition occurred.

\section{B. Experiment}

\section{Materials}

For the experimental studies we used the magnetron sputtering target (Si $n$-type, purity $>99.999 \%$, resistivity $<0.001 \Omega \mathrm{m}$, dimensions: $50 \mathrm{~mm}$ diameter $\times 3 \mathrm{~mm}$ thick), which was purchased from Kurt J. Lesker Company (PA, USA). The Si target was presputtered for 15 minute before deposition on substrates to remove any surface oxide impurities. As substrates for AFM measurements, undoped Si dice with (100) orientation and $5 \mathrm{~mm} \times 5 \mathrm{~mm}$ in size were purchased from MTI Corporation (CA, USA). Carbon-coated grids (400 mesh) were purchased from Ted Pella Inc. (CA, USA), as substrates for TEM/HRTEM analysis.

\section{Deposition of Si NCs}

Size-selected monodispersed Si NCs were synthesized using a magnetron-sputtering inert gas-condensation cluster beam source attached to an inline, quadrupole mass filter (QMF) [Mantis Deposition Ltd.]. Si NC growth was controlled by varying the deposition parameters including magnetron power (dc), aggregation length, gas pressure, and gas flow. We observed that from all the parameters influencing the growth, the Ar/He gas flow rate was the most significant. In this experiment, both $\mathrm{Ar}$ and $\mathrm{He}$ gas flows were varied, whereas the DC magnetron power, aggregation zone length, and deposition times were kept fixed at $90 \mathrm{~W}, 125 \mathrm{~mm}$, and $10 \mathrm{~min}$, respectively.

For the samples described in this paper, we prepared $\mathrm{Si} \mathrm{NCs}$ with three different gas flow conditions, namely, Si-1 (Ar: 80 sccm and He: $40 \mathrm{sccm})$, Si-2(Ar: $80 \mathrm{sccm}$ and He: $10 \mathrm{sccm}$ ) and Si-3 (Ar: $100 \mathrm{sccm}$ and He: $00 \mathrm{sccm}$ ). During deposition, the aggregation zone pressure reading was $3.5 \times 10^{-1} \mathrm{mbar}$ for $\mathrm{Si}-1$ and $\mathrm{Si}-2$, and reading was $4.5 \times 10^{-1}$ mbar for $\mathrm{Si}-3$, respectively. The base pressure in the main deposition chamber was $1.0 \times 10^{-8}$ mbar and the substrate table rotation speed was set at $2 \mathrm{rpm}$.

\section{Characterization}

The Si NCs were characterized by atomic force mMicroscopy (AFM), QMF and transmission electron microscopy (TEM). AFM measurements were performed in a glove-box filled with purified nitrogen to avoid the influence of oxygen and moisture. Both the oxygen and water vapour levels inside the glove-box were kept below $1 \mathrm{ppm}$. The AFM imaging was carried out using the Multimode 8 (Bruker, CA) AFM System equipped with the NanoScope ${ }^{\circledR} V$ controller in peak force tapping mode using triangular silicon-nitride AFM tip (radius $<5 \mathrm{~nm}$, force constant of $0.25 \mathrm{~N} \mathrm{~m}^{-1}, 60 \mathrm{kHz}$ of resonant frequency, Bruker). Height distribution curves were extracted from the AFM images by built-in functions of the scanning probe processor software (SPIP 5.1.8, Image Metrology, Hørsholm, DK). TEM images were obtained using a 300-kV FEI Titan microscope equipped with image spherical aberration corrector. In the TEM, energy dispersive X-ray spectrometry (EDX) was performed with an Oxford X-max system, with an $80-\mathrm{mm}^{2}$ silicon drift detector (SDD) and energy resolution of $136 \mathrm{eV}$.

\section{RESULTS AND DISCUSSION}

\section{A. 4-nm NCs with $\mathrm{SW}$ potential}

We ran 14 individual cases of cooling process of Si NCs from the liquid phase in Ar atmosphere. All the simulations were stopped after $100 \mathrm{~ns}$ when the temperature of the Si 


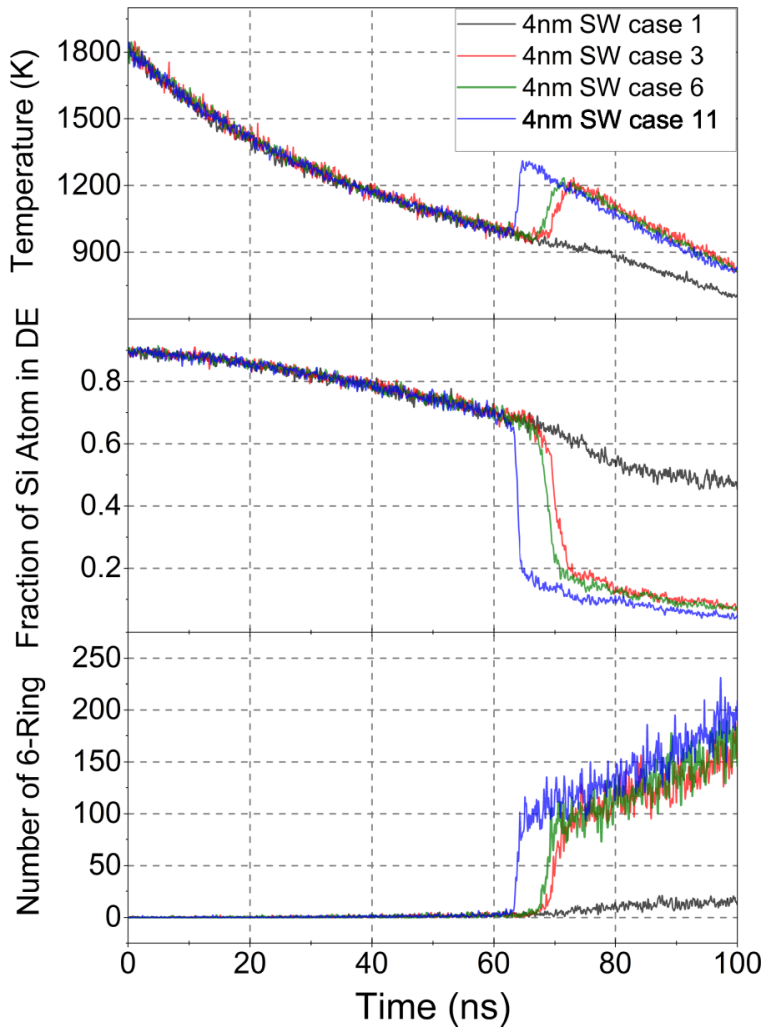

FIG. 2. (Color online) Evolution of the temperature of the $\mathrm{Si}$ nanoclusters obtained in four different simulations (top), the fraction of $\mathrm{Si}$ atoms in a disordered environment (DE) (middle) and the number of primitive sixfold rings (bottom). Simulations cases 3, 6, 11 resulted in crystallization of Si NCs. The "case 1" is shown to represent the results of the runs (including ten other cases not shown here for clarity) in which the Si NCs did not crystallize upon cooling.

nanocluster was about $800 \mathrm{~K}$. Three simulations out of 14 cases showed the crystallization process. To provide quantitative insight into the cooling and crystallization processes, we analyzed the time evolution of the temperature, the fraction of $\mathrm{Si}$ atoms in a disordered environment (DE) and the topological analysis separately for $\mathrm{Si}$ atoms, as shown in Fig. 2. Here we show all cases (cases 3,6, and 11) where crystallization was observed and one typical case (case 1) of a noncrystallized nanocluster. Each simulation run was given a case number to distinguish between the different runs.

In the cases when crystallization of the Si NC had taken place, the phase transition process occurred relatively quickly, within a few nanoseconds. The transition temperature was between 950 and $1000 \mathrm{~K}$, which is far below the melting point of $1350 \mathrm{~K}$ for this size nanocrystals. (See the detailed data in Appendix B.) The temperature curve shows a sharp increase from 1000 to $1300 \mathrm{~K}$ in the case 11 (blue line), indicating a clear latent heat release at $63 \mathrm{~ns}$, which is consistent with the structure and the topological curves. We notice that there are several differences between the simulation case 11 and the cases 3 and 6. The final structure of Si nanocluster in case 11 is more ordered than in the cases 3 and 6 , and the phase transition is more abrupt. All three employed ways of analysis consistently indicate that: the larger the latent heat release, the more complete transition in the nanoclusters is observed. (i)

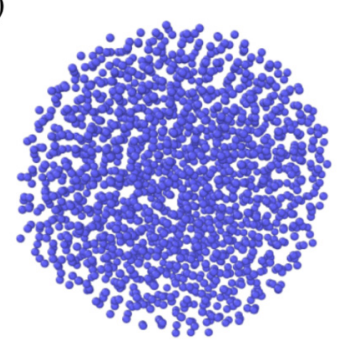

(iii)

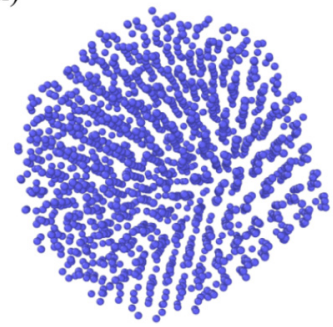

(ii)

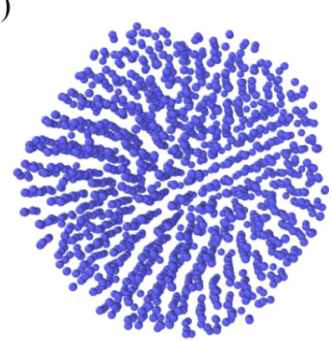

(iv)

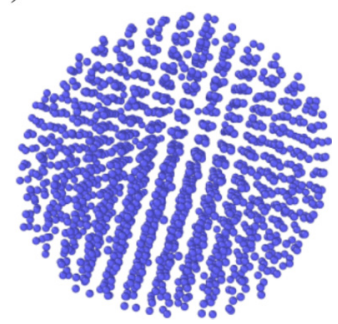

FIG. 3. (Color online) The final structures obtained in the simulations: (i) amorphous in case 1; (ii) and (iii) polycrystalline in cases 3 and 6; (iv) single crystalline in case 11.

The final structures of the four cases are shown in Fig. 3. The structures in the cases 3 and 6 have several nanograins growing in different crystallographic directions.

The polycrystallinity of the final structure can be easily overlooked by the conventional analysis methods, such as radial distribution function (RDF). As shown in Fig. 4, the polycrystallized cases 3 and 6 have an insignificant difference in the rdf from the cases where the structures of Si NCs turned in a single-crystal phase (the cases 11).

The polycrystalline structure can appear due to two reasons: (i) more than one seed starts to grow at the same time; or (ii) diamond and wurtzite structure coexist in the cluster. Since the SW potential has cutoff radius $3.771 \AA$, the diamond and wurtzite structures have the same potential energy at $0 \mathrm{~K}$, since the atoms in these structures have a different local neighborhood only in their third nearest-neighbor position. At high temperature, thermal vibration can bring the third

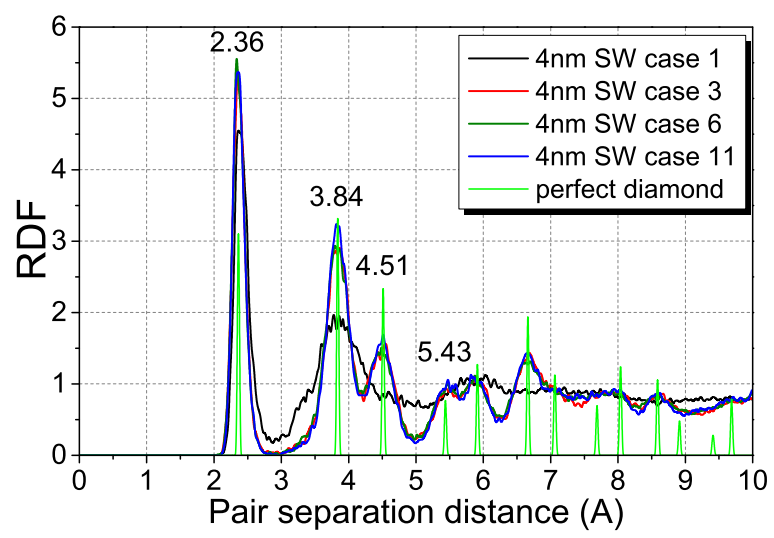

FIG. 4. (Color online) Radial distribution function of the structure after cooling for four simulation cases and reference peak of the perfect Si bulk diamond structure. 
TABLE I. Fraction of surface atoms for ideal crystallized Si NCs of different size.

\begin{tabular}{lrrr}
\hline \hline $\begin{array}{l}\text { Diameter } \\
(\mathrm{nm})\end{array}$ & $\begin{array}{c}\text { Total number } \\
\text { of atoms }\end{array}$ & $\begin{array}{c}\text { Number of } \\
\text { surface atoms }\end{array}$ & $\begin{array}{c}\text { Fraction of } \\
\text { surface atoms }\end{array}$ \\
\hline 3.0 & 702 & 343 & 0.489 \\
3.5 & 1126 & 496 & 0.440 \\
4.0 & 1677 & 647 & 0.386 \\
4.5 & 2383 & 833 & 0.3496 \\
5.0 & 3275 & 1052 & 0.321 \\
6.0 & 5638 & 1517 & 0.269 \\
7.0 & 8970 & 2089 & 0.233 \\
9.0 & 19084 & 3550 & 0.186 \\
12.0 & 45263 & 6482 & 0.143 \\
20.0 & 209320 & 18326 & 0.088 \\
\hline \hline
\end{tabular}

nearest-neighbor inside the cutoff radius to make the wurtzite structure less energetically favorable. If any wurtzite structure exists in the Si NCs, the difference must be observed in the third nearest-neighbor position in the rdf structure. In order to identify the structure, we compared the radial distribution functions of nanoclusters with perfect silicon diamond structure. As shown in Fig. 4, the peaks had good match with the diamond structure. This indicates that the polycrystalline structure grows from multiple seeds rather than the coexistence of diamond and wurtzite structure.

\section{B. 9-nm NCs with SW potential}

We simulated a larger cluster to examine the dependence of the crystallization process on the size and the fraction of surface atom as well as the effect of the cooling rate. If the polycrystalline structure in 4-nm clusters is grown from multiple seeds, these seeds could be identified more clearly in the 9-nm clusters. Table I shows the fraction of surface atoms for Si NCs of different size. Here, we can see that the 9-nm size of the particles has a reasonable combination of the manageable amount of atoms and low fraction of surface atoms (less than half of that for 4-nm nanocluster). In this group of simulations, we applied two cooling rates 20 and $30 \mathrm{~K} / \mathrm{ns}$. With $20 \mathrm{~K} / \mathrm{ns}$ cooling rate, four simulations cases 5, 8, 10, and 11, out of 11 cases in total, went through the crystallization process behaving similarly to the $4 \mathrm{~nm}$ Si NCs. With $30 \mathrm{~K} / \mathrm{ns}$ cooling rate, none of the ten simulations showed crystallization. This can be clearly seen in the structural and topological analyses shown in Fig. 5. We note that the transition temperature is between 1050 and $1100 \mathrm{~K}$, which is slightly higher than for the 4-nm Si NCs. This size-dependent tendency is well in line with the experimental results [49], where the crystallization temperature of the 4- and 8-nm Si cluster were found to be 1073 and $1173 \mathrm{~K}$, respectively (in comparison, the crystallization temperature of bulk $\mathrm{Si}$ is $1250 \mathrm{~K}$ [19]). The difference in crystallization temperatures can be explained by the size effect, similarly to the size dependence of the melting point found for different nanometric crystals [50-52]. The probability of phase transition is related to the cooling rate. From the present results, we find that the critical cooling rate is between 20 and $30 \mathrm{~K} / \mathrm{ns}$.

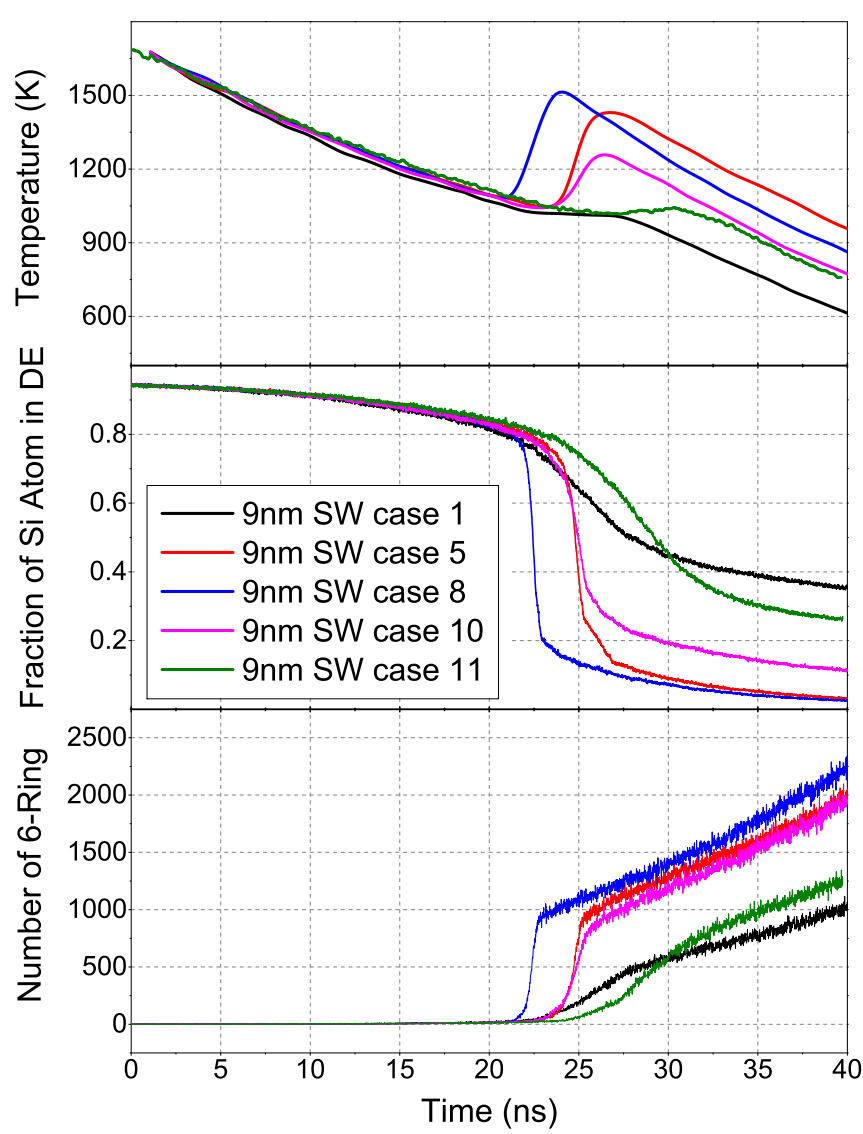

FIG. 5. (Color online) Evolution of the temperature of Si nanocluster (top), the fraction of $\mathrm{Si}$ atoms in a disordered environment (DE), and the number of primitive 6 ring (bottom). Simulation cases $5,8,10$, and 11 showed crystallization. Simulation case 1 is representative of the other noncrystallized simulations, which show a similar evolution.

Topological analysis was used to reveal the positions of seeds of crystallinity. In the simulation case 10, at least three individual crystallites started to grow separately as shown in Fig. 6. The previous estimations of the critical size of a crystalline nucleus at $0.75 T_{m}$ gave a value of 175 atoms [19]. Our results show clearly that for such a small phase, assumption of a spherical seed does not hold any more.
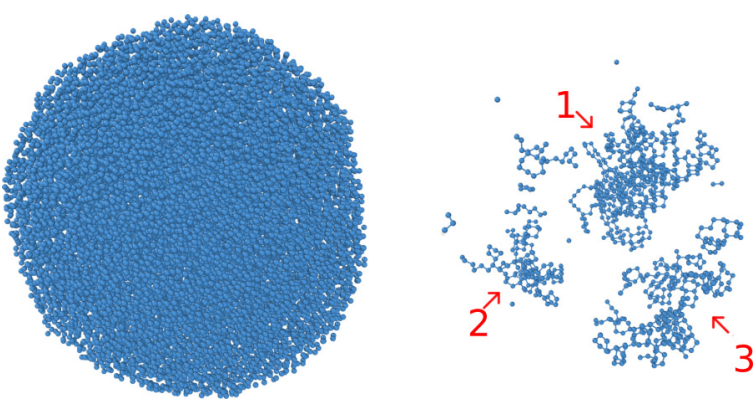

FIG. 6. (Color online) Visualization from topological analysis of the simulation case 10: original cluster (left) and crystallized region (right) at $24.3 \mathrm{~ns}$. Three separate crystallites are identified (red arrows), indicating that polycrystalline structure is grown from the several nuclei of random shapes. 


\section{4-nm NCs with Tersoff potential}

To verify that the results obtained with the SW potential are not artefacts of a specific interatomic potential, we also performed the same simulations for the 4-nm Si NC with the T-III potential. This potential has a known feature of giving a melting point of $2400 \mathrm{~K} \mathrm{[40],} \mathrm{which} \mathrm{is} \mathrm{much} \mathrm{higher} \mathrm{than} \mathrm{the}$ experimental value of $1680 \mathrm{~K}$ [40]), must be taken into account in these series of simulations. To make the results comparable, we increased the initial temperature of Si NCs from 2000 to $2600 \mathrm{~K}$, amounting to about equal homologous temperature. To keep simulations within the reasonable time scale, we also applied different cooling rates from 20 to $60 \mathrm{~K} / \mathrm{ns}$. We controlled the cooling rates by using a different number of $\mathrm{Ar}$ atoms: 200, 150,100, and 50 Ar atoms in $6 \mathrm{~nm} \times 6 \mathrm{~nm} \times 6 \mathrm{~nm}$ box. The cooling process of the liquid Si NCs simulated with the T-III potential shows a very different behavior compared to that obtained with the SW potential. In these simulations we observe that the fraction of $\mathrm{Si}$ atom in a disordered environment starts to decrease at very high temperature $(2600 \mathrm{~K})$. As shown in Fig. 7, within the first $5 \mathrm{~ns}$, the fraction has already dropped from 0.8 to 0.55 , which was never seen in the simulations with the SW potential.

The evolution of the structure factor and the number of the six rings is quite smooth, indicating that no abrupt phase transition happens during the cooling process. At the end of the simulations, the Si NCs form a glasslike structure with short-ranged ordered matrix in the cases with 200, 150, and $100 \mathrm{Ar}$ atoms. In the case of $50 \mathrm{Ar}$ atoms (very low cooling rate), however, the visual inspection of the structures reveals clear crystal planes similar to those observed with the SW potential, as show in Fig. 8(a). In order to validate the result and examine the exact temperature of the crystallization, we ran six more simulations for the case of the $50 \mathrm{Ar}$ atoms with the different initial temperatures of the Si NC, from 2000 to $1000 \mathrm{~K}$. In this group of simulations, as shown in Fig. 9, the Si NC, while cooling from $2000 \mathrm{~K}$, goes through an abrupt phase transition at $1700 \mathrm{~K}$, arriving at a single crystal structure seen in Fig. 8(b). We calculated the ratio of the crystallization temperature $T_{\text {tr }}$ to the bulk melting point $T_{m}$ for both potentials in Table II and found that this ratio does not depend much on the potential (being about 0.7 in both cases) and can be referred as an intrinsic property of the nanoscale Si material. The cooling rate criterion for T-III Si NCs is below $20 \mathrm{~K} / \mathrm{ns}$.

Based on our results, we draw the conclusion that the SW and T-III consistently indicate that the cooling of Si NCs from the temperatures above the critical crystallization temperature (after the aggregation zone in the experiments) with a certain critical rate may result in a crystal (single or poly-) structure of the deposited Si NCs. Some details, such as the crystallization temperature and the rate, may depend on the potential in use.

\section{Experimental growth and deposition of Si NCs}

To demonstrate the very good control over NC size of the magnetron-sputter inert gas-condensation method, three varieties of deposited clusters (experiment sets $\mathrm{Si}-1, \mathrm{Si}-2$, and Si-3) were observed by AFM and TEM; the images taken showcase the dependence of both cluster size and crystallinity on the $(\mathrm{Ar} / \mathrm{He})$ gas flow rate. Figure 10(a) shows AFM images of the $\mathrm{Si}$ NCs (Si-1, Si-2, and $\mathrm{Si}-3$ ) deposited on

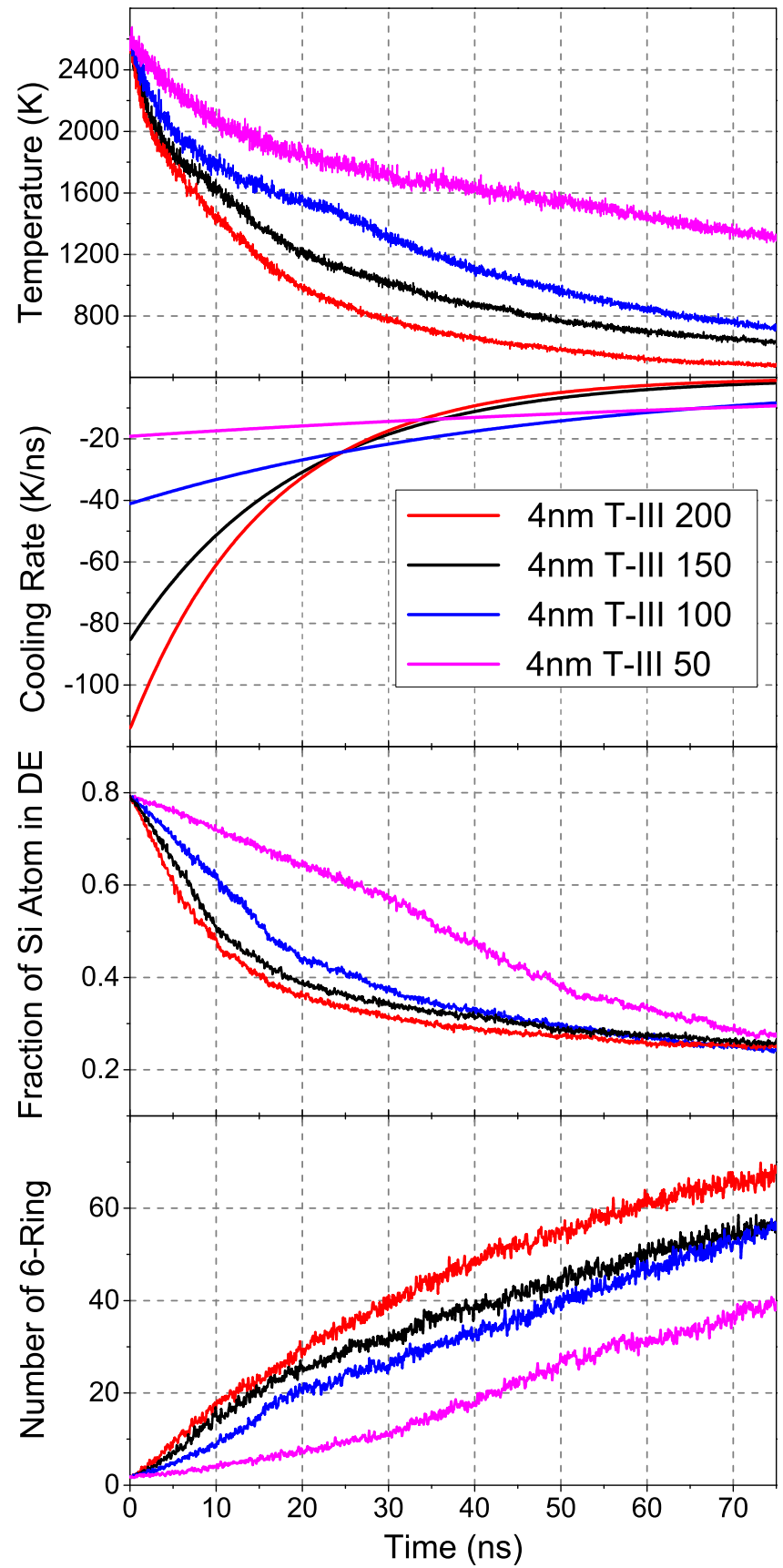

FIG. 7. (Color online) Evolution of the temperature (first) and the cooling rate (second) of $\mathrm{Si}$ nanocluster, the fraction of $\mathrm{Si}$ atoms in a disordered environment (DE) (third) and the number of primitive 6-ring (fourth).

the Si substrate. The size distribution of Si NCs is shown in Figure 10(b). Their average size calculated from the AFM images are $2.5 \pm 0.8,5.2 \pm 1.2$, and $8.2 \pm 2.0 \mathrm{~nm}$ for $\mathrm{Si}-1$, $\mathrm{Si}-2$, and $\mathrm{Si}-3$, respectively. It was found by AFM observation that the average size of $\mathrm{Si} \mathrm{NCs}$ was reduced when the $\mathrm{He}$ gas flow rate was increased from 0 to $40 \mathrm{sccm}$. The average size measured by AFM was in good agreement with the QMF selected sizes [Fig. 10(c)] for $\mathrm{Si}-1, \mathrm{Si}-2$, and $\mathrm{Si}-3$ clusters. In the $\mathrm{Si}-1$ deposition, large number densities and small $\mathrm{Si}$ $\mathrm{NC}$ sizes with narrow size distribution were obtained. In the 

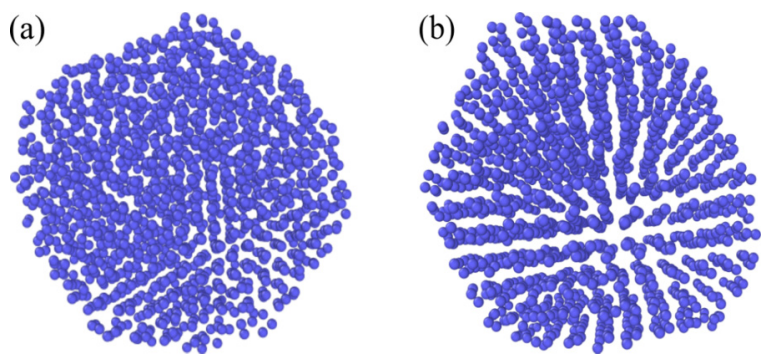

FIG. 8. (Color online) The final structure of the Tersoff potential simulation cases (a) $50 \mathrm{Ar}$, initial temperature $2600 \mathrm{~K}$; (b) $50 \mathrm{Ar}$, initial temperature $2000 \mathrm{~K}$.

Si-2 deposition, relatively lower number densities and larger silicon nanoparticle sizes, with narrow size distribution, were obtained. Finally, in the Si-3 deposition, relatively wide size distributions were encountered due to the absence of He flow and higher Ar flow rates, which favors NC growth [34].

Figure 11 shows the TEM images of Si NCs, produced using $\mathrm{Si}-1, \mathrm{Si}-2$, and $\mathrm{Si}-3$ deposition conditions, respectively. The cluster size ranged from 2 to $18 \mathrm{~nm}$, with the bigger clusters corresponding to the large-size tails of the Si-3

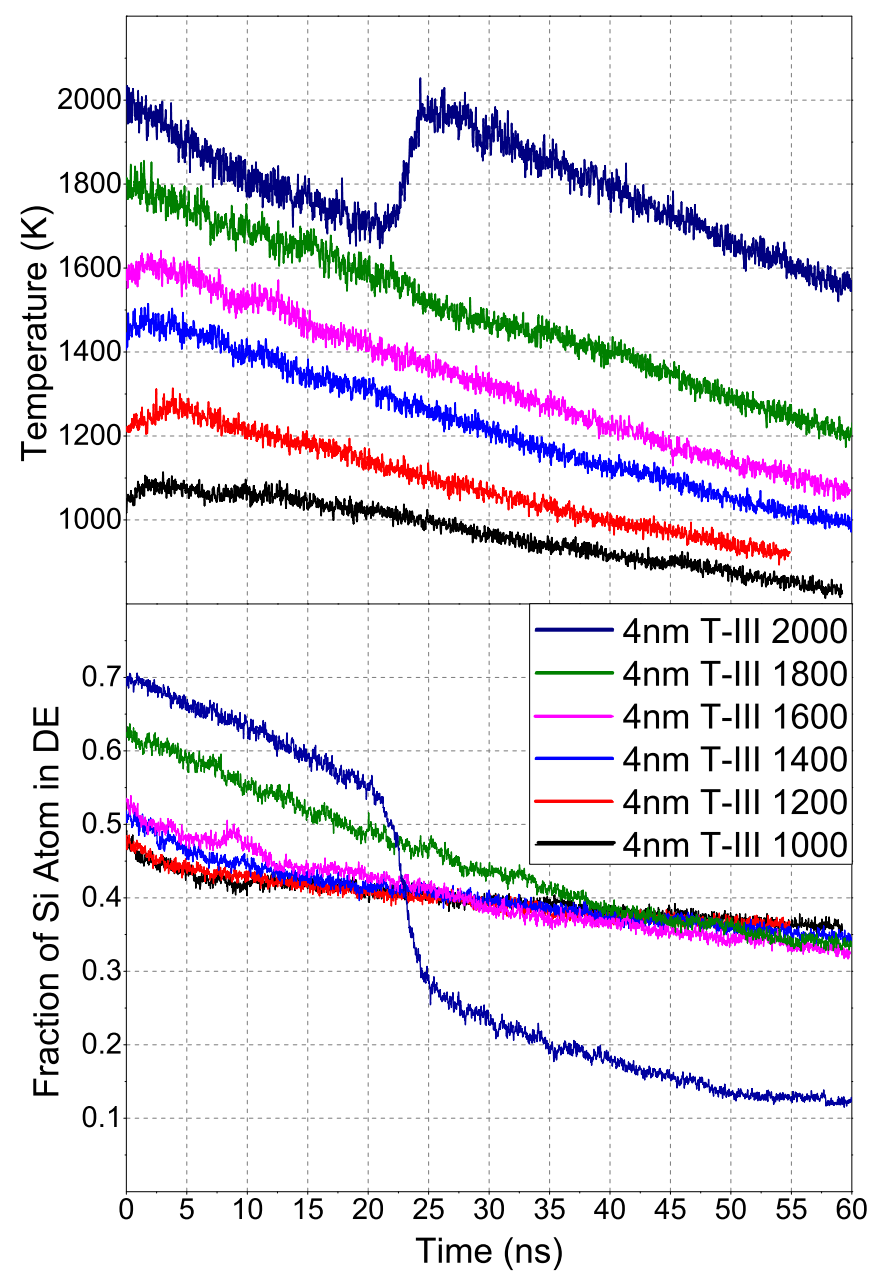

FIG. 9. (Color online) Evolution of the temperature of Si NC (top) and the fraction of $\mathrm{Si}$ atoms in a disordered environment (DE) (bottom).
TABLE II. Ratio of the transition temperature to the melting point for both potentials. For the melting point of the $4 \mathrm{~nm} \mathrm{Si}$ NCs, see Appendix B.

\begin{tabular}{lccccc}
\hline \hline Potential & $T_{m}($ bulk $)$ & $T_{m}(4 \mathrm{~nm})$ & $T_{\text {tr }}$ & $T_{\text {tr }} / T_{m}($ bulk $)$ & $T_{\text {tr }} / T_{m}(4 \mathrm{~nm})$ \\
\hline SW & $1691 \mathrm{~K} \mathrm{[53]}$ & $1450 \mathrm{~K}$ & $1000 \mathrm{~K}$ & 0.594 & 0.690 \\
T-III & $2589 \mathrm{~K} \mathrm{[54]}$ & $2175 \mathrm{~K}$ & $1700 \mathrm{~K}$ & 0.657 & 0.782 \\
\hline \hline
\end{tabular}

distributions of Figs. 10(b) and 10(c). The resulting spherical nanoparticles were monodisperse and it was found that they were in accordance with the results obtained from AFM and QMF. An energy-dispersive spectrometry(EDS) analysis was performed for the silicon nanoparticles on the selected region indicated in Fig. 12. Silicon content was confirmed, with peak $\mathrm{x}$-ray emission intensity at $1.8 \mathrm{keV}$, corresponding to the $\mathrm{Si}-\mathrm{K} \alpha$ line. Oxygen was also detected, indicating that some surface oxidation of the particles has occurred during storage/transfer.

Figures 13(a) and 13(b) show high-resolution TEM (HRTEM) images of a 9-nm and a 16-nm NC synthesized under the Si-3 deposition conditions. The corresponding fast Fourier transform (FFT) analysis of the HRTEM image regions indicate that the particles have a polycrystalline silicon structure. The main reflections (111), (220), and (311) are indexed in the figures (JCPDS card No. 27-1402), and correspond to the $\mathrm{Si}$ diamond structure. Both findings are in excellent agreement with the aforementioned simulation results under the SW potential.

\section{E. Comparison of simulations and experiments}

To understand how far the condition, which we are able to simulate by MD differs from the experimental condition, we attempt to bridge the results obtained in simulations and experiments. In Table III, we list some essential parameters which may influence the crystallization process of the Si NCs.

The major uncertainty in the comparison is how the temperature evolution takes place in the nanocrystals in the aggregation zone and in the deposition chamber. Although MD simulations are capable of describing the process of thermodynamic equilibration of a nanocluster quite accurately, the phase transitions liquid-amorphous-crystalline depend on how fast the temperature is changing in the system. To analyze this, we developed an analytical model to estimate the temperature evolution in the Si NCs. First in the aggregation zone, the temperature evolves as a balance between the cooling by atoms of the Ar atmosphere [the first term in Eq. (3)] and the heating due to $\mathrm{Si}$ atoms coalescence [the second term in the Eq. (3)], as shown in Fig. 14:

$$
\begin{aligned}
\frac{d T(t)}{d t}= & \frac{1}{3 k_{B} N(t)}\left[k \pi r_{c}^{2} v_{d} \rho_{\mathrm{Ar}}\left(T_{\mathrm{Ar}}-T(t)\right)\right. \\
& \left.+2 \pi r_{c}^{2} v_{d} \rho_{S i} E_{\mathrm{co}}\right]
\end{aligned}
$$

where $E_{\mathrm{co}}$ is the released potential energy of the single Si atom coalescence, $N(t)$ is the number of the atoms in the NC, $r_{c}$ is the radius of the $\mathrm{NC}, v_{d}$ is the drift velocity of the $\mathrm{NC}, \rho_{\mathrm{Ar}}$ and $\rho_{S i}$ are the number densities of the $\mathrm{Ar}$ and $\mathrm{Si}$ atoms in the gas phase, respectively, $T_{\mathrm{Ar}}$ is the temperature of the Ar atoms, $k$ is the energy exchange constant, and $k_{B}$ is the Boltzmann 

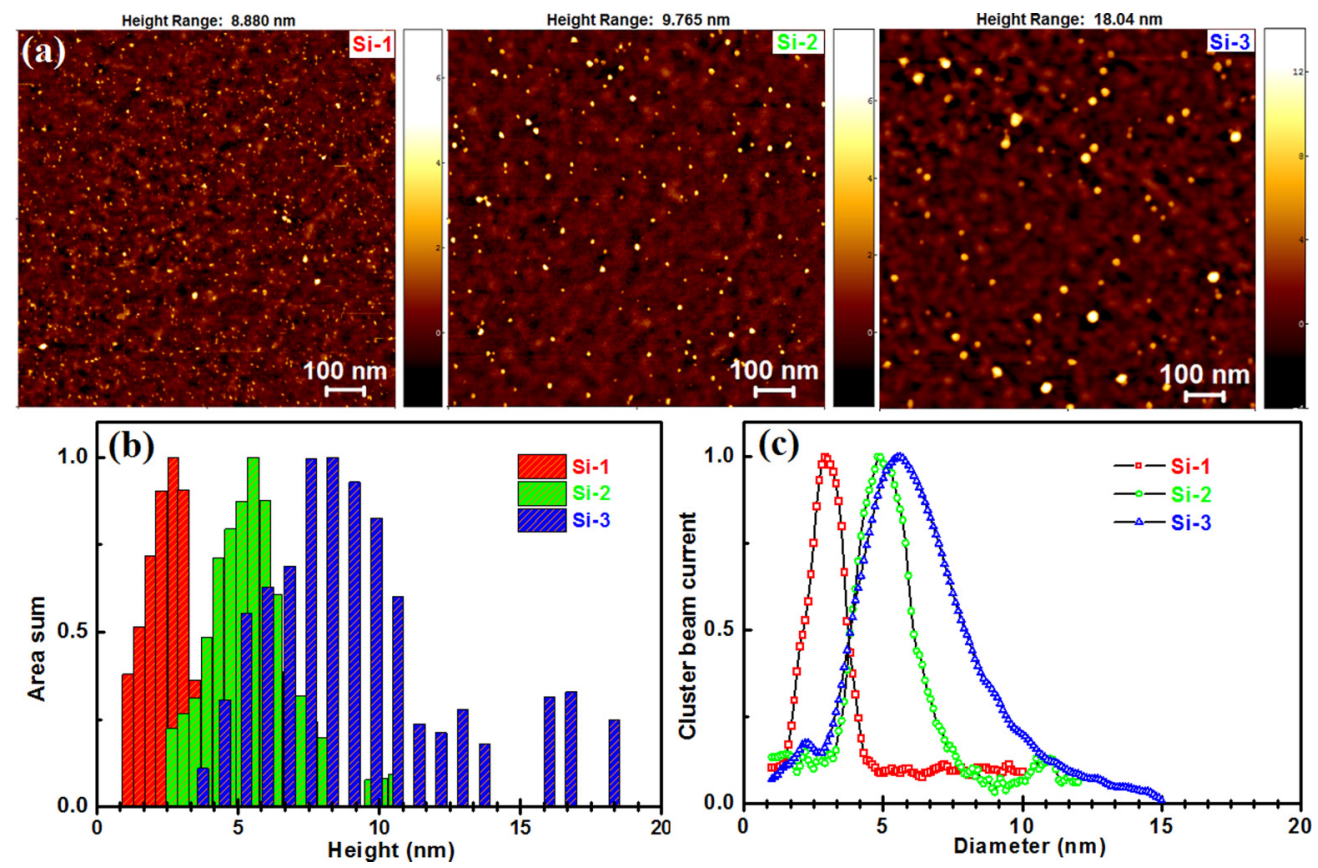

FIG. 10. (Color online) (a) High-resolution AFM topography images, (b) AFM height distributions, and (c) quadruple mass filter (QMF) profiles for $\mathrm{Si}$ nanoparticles deposited using varying $\mathrm{Ar}$ and $\mathrm{He}$ gas flow ( $\mathrm{Si}-1, \mathrm{Si}-2$, and $\mathrm{Si}-3$ ) at a deposition power of $90 \mathrm{~W}$ and time of 10 minute.

constant. For details of derivation and solution of Eq. (3), see Appendix A.

The solution presented in Fig. 14 is given for the initial temperature $3000 \mathrm{~K}$ and the initial diameter $4 \mathrm{~nm}$ of a Si NC. In this solution, we see that the final temperature depends only on the ratio of the number density of the Si and Ar atoms $\rho_{\mathrm{Si}} / \rho_{\mathrm{Ar}}$. It is clear that the equilibrium temperature of the $\mathrm{NC}$ is very sensitive to the $\rho_{\mathrm{Si}} / \rho_{\mathrm{Ar}}$ ratio. If the $\rho_{\mathrm{Si}} / \rho_{\mathrm{Ar}}$ ratio is below about $1 / 20$ in the aggregation zone, the steady-state temperature of the NC will not exceed the crystallization temperature (cf. Fig. 9). Hence there is a critical $\rho_{\mathrm{Si}} / \rho_{\mathrm{Ar}}$ ratio allowing the $\mathrm{Si}$ $\mathrm{NCs}$ to be heated over the crystallization temperature during the condensation process.

The temperature evolution in the deposition chamber can be described by only the cooling term in Eq. (3), as there is no aggregation of NC's in this stage. Since the number density of the Si atoms vanished to zero, the size of the Si NCs is a constant. The NCs will naturally cool down to the atmosphere temperature $300 \mathrm{~K}$. The cooling rates of the NCs depend on the Ar collision rate with the Si NC, $n_{\mathrm{co}}$, which is expressed here in terms of the number density of Ar atoms available for collision with the Si NC of a certain size, and the size of the NCs [see Eq. (A3)]. As shown in Fig. 15, the larger-sized NCs have a lower cooling rate. Since the crystallization is a stochastic process, the slower cooling provides longer times for a phase transition to take place, as the NC stays longer at the crystallization temperature during the cooling process. This may explain the crystallization found in large 9-nm and 16-nm $\mathrm{NCs}$ in the experiments. The cooling rate in the experimental condition was between 50 and $10 \mathrm{~K} / \mu \mathrm{s}$, as shown in Fig. 15, which is three orders of magnitude smaller than that in the simulations.

Since slower cooling rates generally promote crystallization, and the simulations also showed crystallization even though the cooling rates in the simulations are much higher than those in the experiments, the cooling rate alone cannot explain why experiment sets $\mathrm{Si}-1$ and $\mathrm{Si}-2$ did not show crystallization, while $\mathrm{Si}-3 \mathrm{did}$. The reason for the differences

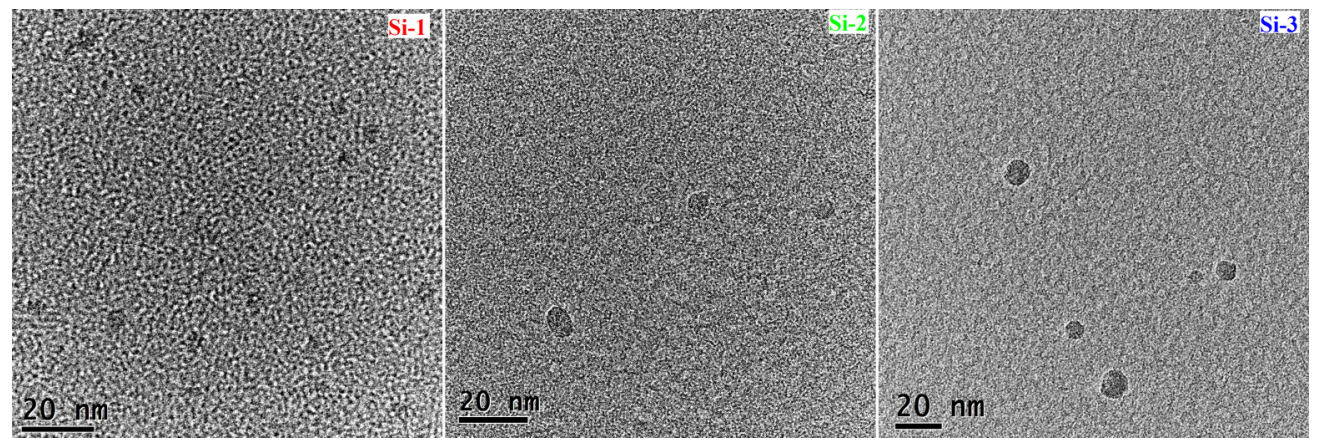

FIG. 11. (Color online) TEM images for Si nanoparticles deposited using varying Ar and $\mathrm{He}$ gas flows (Si-1, Si-2, and $\mathrm{Si}-3$ ) at a deposition power of $90 \mathrm{~W}$ and time of 10 minutes. 


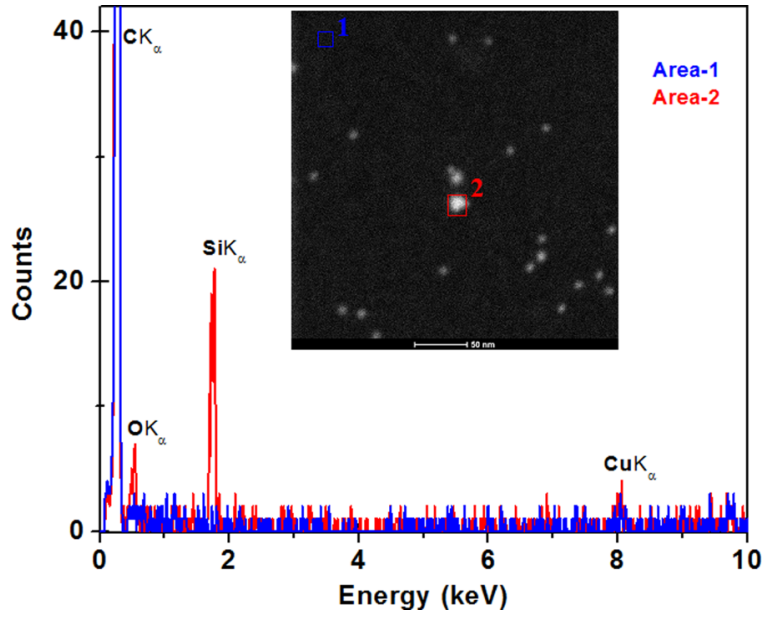

FIG. 12. (Color online) EDS spectra from corresponding selected area as shown inset high-angle annular dark field scanning transmission electron microscopy (HAADF-STEM) image of silicon nanoparticles. The $\mathrm{Cu}$ and $\mathrm{C}$ peaks are attributed to the carbon-coated copper grid used in TEM.

in the experimental groups is most likely related to the temperature reached in the condensation process. Unfortunately, calculation of the exact temperature of the growing cluster in the experimental chamber would require more precise data on the magnetron plasma properties and partial gas pressures and flow velocities than what is currently available. One thing which is clear, however, is that the initial temperature of the $\mathrm{Si}$ atoms is high since they originate from a sputtering process, and sputtered particles typically have emission kinetic energies
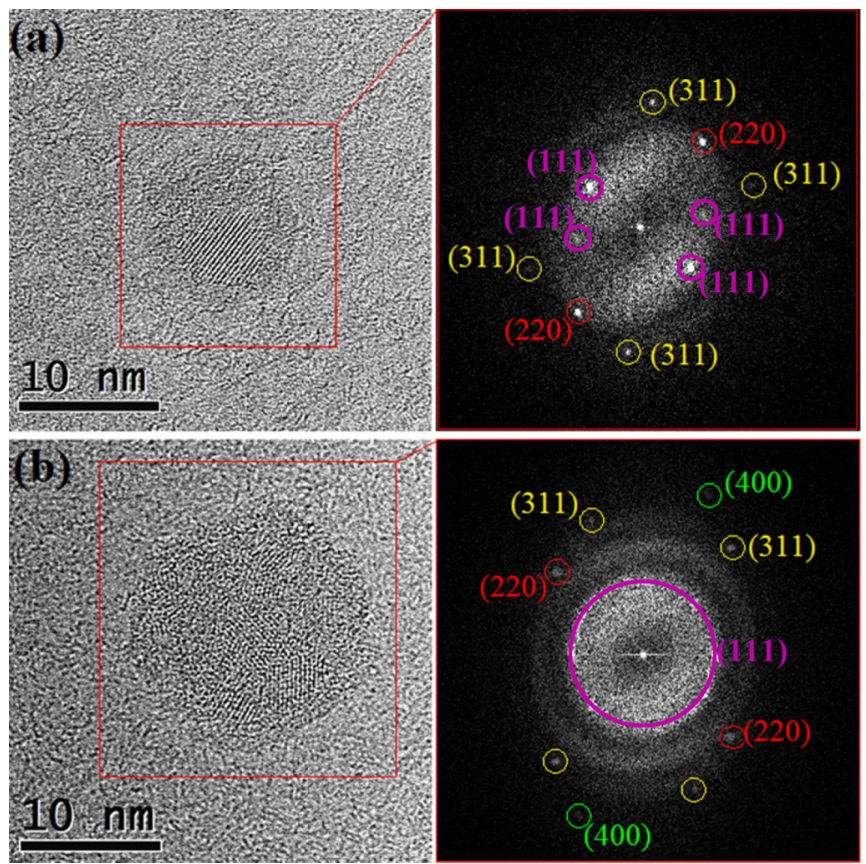

FIG. 13. (Color online) HRTEM images with corresponding FFTs from the (a) 9-nm and (b) 16-nm nanoparticle at Si-3 deposition condition. FFT with clear spot pattern from red boxed region shows a characteristic of crystalline silicon lattice structure.
TABLE III. Important parameters in simulations and experiments. The critical cooling rate in experiment is not available (N.A.), since this quantity is not measurable in the current setup.

\begin{tabular}{lccc}
\hline \hline Simulations & $\begin{array}{c}\text { Cluster } \\
\text { diameter }(\mathrm{nm})\end{array}$ & $\begin{array}{c}\text { Pressure } \\
(\text { Bar })\end{array}$ & $\begin{array}{c}\text { Critical cooling } \\
\text { rate }(\mathrm{K} / \mathrm{ns})\end{array}$ \\
\hline SW & 4 & 0.87 & 20 \\
SW & 9 & 33 & 20 \\
T-III & 4 & 11 & 15 \\
& Cluster & Pressure & $\begin{array}{c}\text { Critical cooling } \\
\text { Experiments }\end{array}$ \\
diameter $(\mathrm{nm})$ & $(\mathrm{Bar})$ & rate $(\mathrm{K} / \mathrm{ns})$ \\
$\mathrm{Si}-1$ & 2.5 & $3.5 \times 10^{-4}$ & N.A. \\
$\mathrm{Si}-3$ & 5 & $3.5 \times 10^{-4}$ & N.A. \\
\hline \hline
\end{tabular}

in the $\mathrm{eV}$ range. After the condensation begins, however, the clusters will cool down depending on the $\rho_{\mathrm{Si}} / \rho_{\mathrm{Ar}}$ ratio and flow velocity. The presence of $\mathrm{He}$ in experiment set $\mathrm{Si}-1$ and $\mathrm{Si}-2$ increases the flow velocity and reduces the $\mathrm{Si} / \mathrm{Ar}$ ratio, which decreases the temperature the Si cluster reaches (note from Figure 11 that the cluster produced in experiment sets $\mathrm{Si}-1$ and $\mathrm{Si}-2$ are also smaller, which is consistent with $\mathrm{Si}$ undergoing less collisions). In experiment set $\mathrm{Si}-3$, with no $\mathrm{He}$, the clusters can apparently reach a high enough temperature that recrystallization becomes possible.

In simulations, we also see that both the initial temperature and the cooling rate play important roles in the crystallization process as shown in Figs. 7 and 9. From here we conclude that the size dependence of crystallization process apparent in experiment, is a result of the combination of how high an NC temperature is reached in the condensation process, and the low cooling rate (about $20 \mathrm{~K} / \mu$ s) in the deposition chamber.

\section{CONCLUSION}

The magnetron-sputter inert gas-condensation method is capable of producing Si NCs with good control over NC size and crystallinity. Utilizing it under various inert gas conditions, a series of polycrystalline Si NC's were synthesized. To comprehend the crystallization mechanism, we simulated freestanding NCs, under inert gas temperature control, a natural

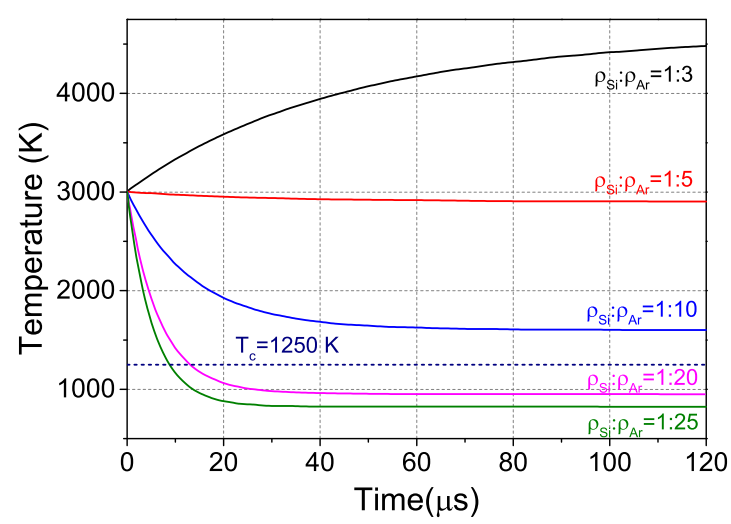

FIG. 14. (Color online) Temperature evolution in the aggregation zone from the analytical model. 


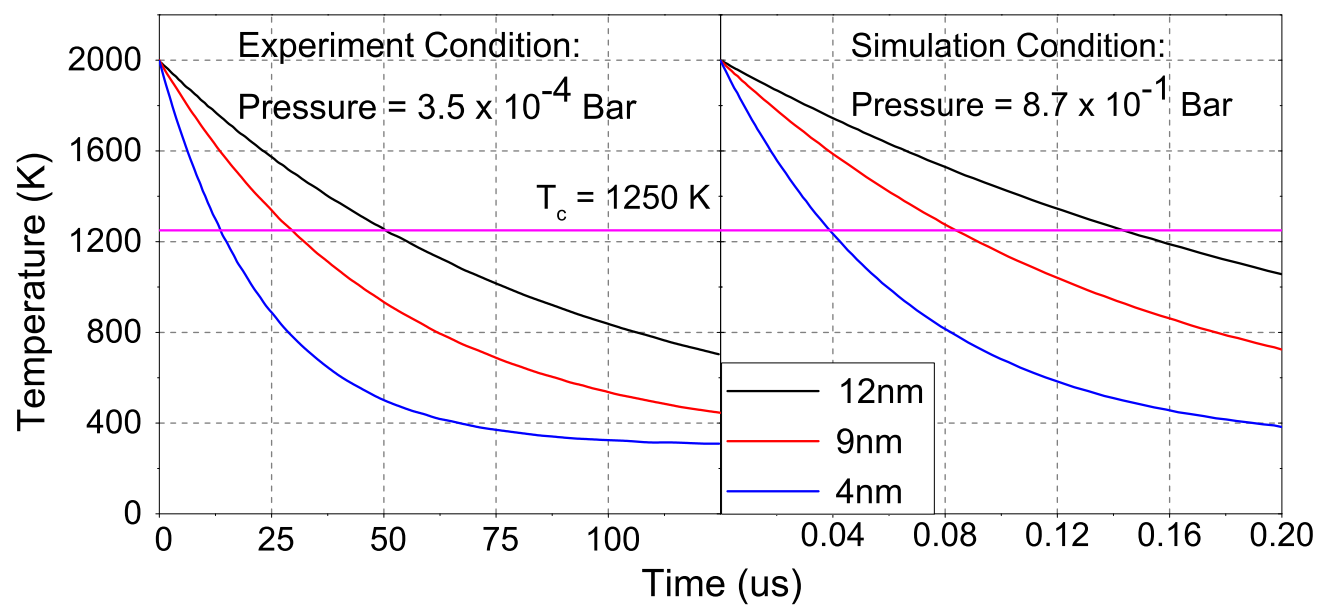

FIG. 15. (Color online) Temperature evolution under experiment condition (left) and simulation condition (right).

and realistic method to simulate free-standing nanoclusters. We ran more than ten simulations at each condition to get relatively good statistics for the observed behavior, using two interatomic potentials, namely the SW and T-III. The crystallization temperature of the SW Si NC is dependent on the size of the system, whereas the probability of crystallization is dependent on the initial temperature of the formed cluster and the cooling rate. It is the first time that the nanopolycrystalline structure has been observed in the SW Si NC system, and agrees with our experimentally grown NCs. The evidence shows clearly that nano-polycrystallites grow from separate nuclei. The results obtained with the two potentials, SW and T-III, are consistent, which lead to the conclusion that the crystallization of tetrahedrally bonded semiconductors is a general process that can be expected to occur in the insert gas condensation method.

\section{ACKNOWLEDGMENTS}

This study was supported by the doctoral programme MATRENA. We also gratefully acknowledge the grants of computer time by the CSC, the Finnish IT center of science. We also thank Antti Kuronen and Morten Nagel for useful discussions.

\section{APPENDIX A: MODEL FOR COOLING RATE}

\section{Inert gas pressure and cooling rate}

With the use of scaled coordinates, one can evaluate the internal pressure in NVT ensemble by

$$
\begin{aligned}
P^{(\text {int })} & =k_{B} T \frac{\partial}{\partial V} \ln Q(N, V, T) \\
& =\frac{N k_{B} T}{V}+\frac{1}{3 V} \sum_{i}\left\langle\mathbf{r}_{i} \cdot \mathbf{f}_{i}\right\rangle_{N V T},
\end{aligned}
$$

where we can insert temperature definition $3 N k_{B} T=$ $\left\langle\sum_{i} \mathbf{p}_{i}^{2} / m_{i}\right\rangle_{N V T}$ to obtain

$$
P(\Gamma(t))=\frac{1}{3 V} \sum_{i}\left(\frac{\mathbf{p}_{i}^{2}}{m_{i}}+\mathbf{r}_{i} \cdot \mathbf{f}_{i}\right) .
$$

One can estimate the cooling rate of the NC from the number density of inert gas $\rho_{\text {gas }}=N_{\text {gas }} /\left(V_{s}-V_{c}\right)$, where $V_{s}$ and $V_{c}$ are the volume of the simulation cell and the volume of cluster respectively. We regard the NC as a sphere with radius $r_{c}$. The $\mathrm{NC}$ has a drift velocity $v_{d}$. The collision rate between the NC and the inert gas is

$$
n_{\mathrm{co}}=\pi r_{c}^{2} v_{d} \rho_{\mathrm{gas}},
$$

where $\rho_{\text {gas }}$ can be replaced by the number density of Ar atoms $\rho_{\text {Ar }}$ and $\mathrm{Si}$ atoms in gas phase $\rho_{\mathrm{g}-\mathrm{Si}}$.

Now we have to obtain the average energy transfer per collision $E_{t}$, which is dependent on the masses of atoms $m_{c}$ and $m_{g}$ and the temperature of atoms $T_{c}$ and $T_{g}$. For simplicity, we assume the average transferred energy is due to elastic collision. From Ref. [55], the average transfer is captured by an expression

$$
E_{t}=k\left(T_{g}-T_{c}\right),
$$

the temperature change $\Delta T$ in one collision can be estimated by

$$
\frac{3 n}{2} k_{b} \Delta T=\frac{k}{2}\left(T_{g}-T_{c}\right),
$$

where $n$ is the number of atoms in the cluster, $k$ is the energy exchange constant, which is a function of gas atomic mass, cluster mass and interaction strengths. The cluster temperature after $m$ collisions is given by

$$
T_{c}(m)=\left(T_{c}(0)-T_{g}\right)\left(1-\frac{k}{3 n k_{B}}\right)^{m}+T_{g},
$$

where $m$ can be substituted by $n_{\text {co }} t$. Finally, the cooling rate of cluster at time $t$ is

$$
\frac{d T_{c}(t)}{d t}=\left(T_{c}(0)-T_{g}\right) n_{\mathrm{co}}\left(1-\frac{k}{3 n k_{B}}\right)^{n_{\mathrm{co}} t} \ln \left(1-\frac{k}{3 n k_{B}}\right) .
$$

An example for the Si cluster case (Fig. 16) shows that the temperature of the Si cluster can be fitted perfectly with $\mathrm{t}$ simple function $T(t)=T_{g}+A B^{t}$. By evaluating the slope of the fitting curve, one can estimate the cooling rate as a function of time. As shown in Fig. 16, the cooling rate is about $10 \mathrm{~K} / \mathrm{ns}$ at $50 \mathrm{~ns}$. 


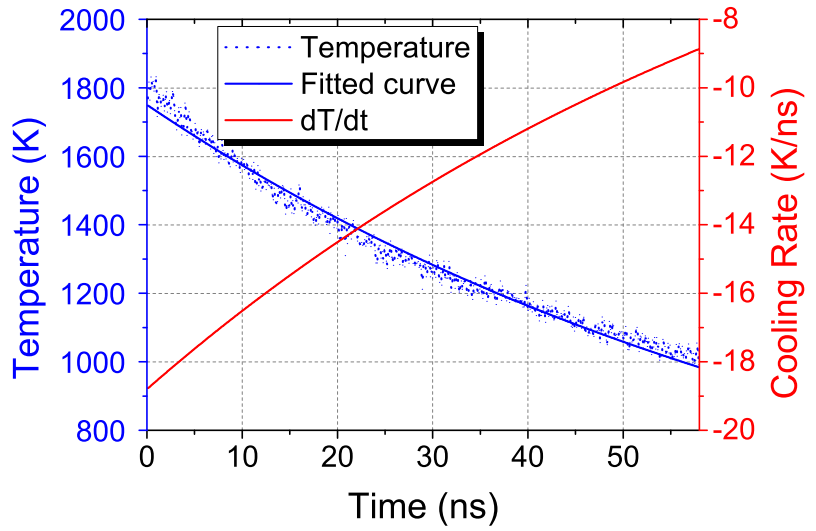

FIG. 16. (Color online) Example of fitted cooling rate of Si NC: 4-nm SW Si nanocluster simulation case 1 . The fitting can only be done before the phase transition occurring to avoid the effect of the latent heat release.

\section{Temperature evolution of NCs in aggregation zone}

We now consider the thermal evolution of a more complex system. A small Si NC flies in a chamber with both Ar and $\mathrm{Si}$ atoms. The NC is cooled down by Ar atoms and heated up by $\mathrm{Si}$ atoms at the same time. The size of the NC increases during the condensation process as the $\mathrm{Si}$ atoms landing on the surface of the NC. The number of the atoms in the NC $N$ can be solved as a function of flying time:

$$
\begin{aligned}
N(t) & =N_{0}+\int_{0}^{t} n_{\mathrm{co}-\mathrm{Si}}(t) d t \\
& =N_{0}+\int_{0}^{t} \pi r(t)^{2} \rho_{\mathrm{Si}} v_{d} d t,
\end{aligned}
$$

where $N_{0}$ is the initial number of the atoms in the cluster and $\rho_{\mathrm{Si}}$ stands for the number density of the $\mathrm{Si}$ atoms in the gas phase. We assume $\overline{V_{0}}$ is the average volume for a single $\mathrm{Si}$ atom. The relation between the radius of the $\mathrm{NC} r_{c}(t)$ and $N(t)$ is

$$
r_{c}=\left(\frac{3 \overline{V_{0}} N(t)}{4 \pi}\right)^{1 / 3}
$$

With the boundary condition $N(t=0)=N_{0}, N(t)$ can be solved as

$$
N(t)=\frac{1}{27}\left[\pi v_{d} \rho_{\mathrm{Si}}\left(\frac{3 \overline{V_{0}}}{4 \pi}\right)^{2 / 3} t+3 N_{0}^{1 / 3}\right]^{3} .
$$

We further assume that each $\mathrm{Si}$ atom which has a collision with the $\mathrm{NC}$, forms bonds and releases potential energy $E_{\text {co }}$ into kinetic energy, so the kinetic energy change of the $\mathrm{NC}$

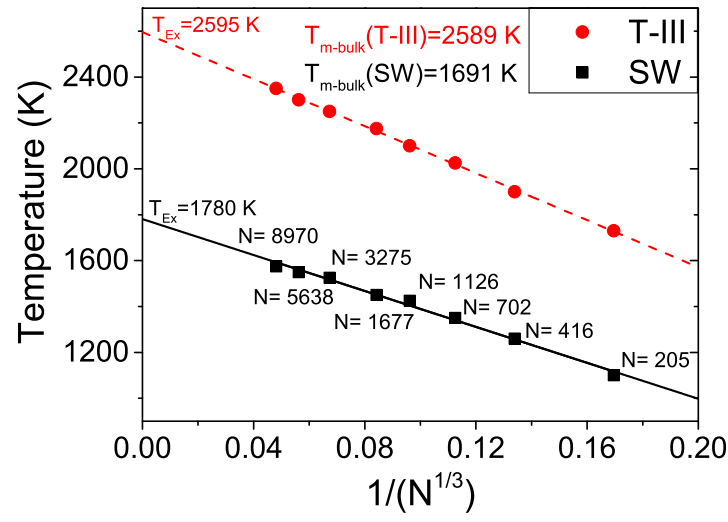

FIG. 17. (Color online) Dependence of melting points on cluster size and potential. The melting points of Si NCs can be linearly fitted to a function of $N^{-1 / 3}$. Extrapolation of linear fitting to bulk $\left(N^{-1 / 3}=0\right)$ is 1779 and $2597 \mathrm{~K}$ for the two potentials, respectively. For bulk Si data see Refs. [53,54].

$\frac{3}{2} k_{B} N(t) d T$ over small $d t$ is

$\frac{3}{2} k_{B} N(t) d T(t)=\frac{k}{2}\left(T_{\mathrm{Ar}}-T(t)\right) n_{\mathrm{co}-\mathrm{Ar}} d t+n_{\mathrm{co}-\mathrm{Si}} E_{\mathrm{co}} d t$.

By substituting $n_{\mathrm{co}-\mathrm{Si}}=\pi r_{c}^{2} v_{d} \rho_{\mathrm{Si}}$ and $n_{\mathrm{co}-\mathrm{Ar}}=\pi r_{c}^{2} v_{d} \rho_{\mathrm{Ar}}$, we obtain

$$
\frac{d T(t)}{d t}=\frac{k \pi r_{c}^{2} v_{d} \rho_{\mathrm{Ar}}\left(T_{\mathrm{Ar}}-T(t)\right)+2 \pi r_{c}^{2} v_{d} \rho_{\mathrm{Si}} E_{\mathrm{co}}}{3 k_{B} N(t)}
$$

and we can further substitute the expressions Eq. (A9) for $r_{c}$ and Eq. (A10) $N(t)$ into the equation. With the boundary condition $T(t=0)=T_{0}$, the final equation can be solved analytically, which gives the NC's temperature as a function of time controlled by the parameters $\rho_{\mathrm{Ar}}, \rho_{\mathrm{Si}}, v_{d}$.

In the deposition chamber, the number density of the $\mathrm{Si}$ atoms $\rho_{\mathrm{Si}}$ vanishes to zero and the size of the Si NCs remains as a constant. By eliminating the heating-up term in Eq. (A12), we have a simple cooling function:

$$
\frac{d T(t)}{d t}=\frac{k \pi r_{c}^{2} v_{d} \rho_{\mathrm{Ar}}\left[T_{\mathrm{Ar}}-T(t)\right]}{3 k_{B} N} .
$$

\section{APPENDIX B: MELTING POINT SIMULATION}

We have performed MD simulations with SW and T-III potentials to evaluate the melting temperature of the Si NCs comprising a maximum of 8970 atoms. The simulations were carried out by heating a cluster from a low temperature to higher temperatures. The temperature increment of each step was $25 \mathrm{~K}$, followed by 500 ps equilibration run. The result has acceptable agreement with a previous study [56,57]. Using the Berendsen thermostat, we have obtained two complete melting curves with the SW and T-III potentials, as shown in Fig. 17.
[1] F. Erogbogbo, K.-T. Yong, I. Roy, R. Hu, W.-C. Law, W. Zhao, H. Ding, F. Wu, R. Kumar, M. T. Swihart, and P. N. Prasad, ACS Nano 5, 413 (2011).
[2] F. Erogbogbo, C.-W. Chang, J. L. May, L. Liu, R. Kumar, W.-C. Law, H. Ding, K. T. Yong, I. Roy, M. Sheshadri, M. T. Swihart, and P. N. Prasad, Nanoscale 4, 5483 (2012). 
[3] N. Sanvicens and M. P. Marco, Trends Biotechnol. 26, 425 (2008).

[4] R. E. I. Schropp, R. Carius, and G. Beaucarne, MRS Bulletin 32, 219 (2007).

[5] J. Choi, J. Cheon, S. Kim, and J. Jang, Displays 26, 137 (2005).

[6] A. H. Mahan, S. P. Ahrenkiel, R. E. I. Schropp, H. Li, and D. S. Ginley, Thin Solid Films 516, 529 (2008).

[7] W. D. Callister, Jr., Materials Science and Engineering, An Introduction, 3rd ed. (Wiley, New York, 1993).

[8] A. V. Granato, Phys. Rev. Lett. 68, 974 (1992).

[9] C. Donati, S. C. Glotzer, P. H. Poole, W. Kob, and S. J. Plimpton, Phys. Rev. E 60, 3107 (1999).

[10] S. C. Glotzer, J. Non-Cryst. Solids 274, 342 (2000).

[11] Z. H. Jin, P. Gumbsch, K. Lu, and E. Ma, Phys. Rev. Lett. 87, 055703 (2001).

[12] L. Pelaz, L. A. Marqués, M. Aboy, J. Barbolla, and G. H. Gilmer, Appl. Phys. Lett. 82, 2038 (2003).

[13] M. Forsblom and G. Grimvall, Phys. Rev. B 72, 054107 (2005).

[14] A. V. Granato, Eur. Phys. J. B 87, 18 (2014).

[15] A. Mattoni and L. Colombo, Phys. Rev. B 78, 075408 (2008).

[16] C. Krzeminski, Q. Brulin, V. Cuny, E. Lecat, E. Lampin, and F. Cleri, Journal of Applied Physics 101, 123506 (2007).

[17] S. M. Nakhmanson and N. Mousseau, J. Phys.: Condens. Matter 14, 6627 (2002).

[18] S. Sastry and C. A. Angell, Nat. Mater. 2, 739 (2003).

[19] P. Beaucage and N. Mousseau, Phys. Rev. B 71, 094102 (2005).

[20] S. Nosé, Mol. Phys. 52, 255 (1984).

[21] W. G. Hoover, Phys. Rev. A. 31, 1695 (1985).

[22] H. C. Andersen, J. Chem. Phys. 72, 2384 (1980).

[23] P. Krasnochtchekov, K. Albe, and R. Averback, Zeitschrift Fur Metallkunde 94, 1098 (2003).

[24] C. Cassidy, V. Singh, F. Djurabekova, K. Nordlund, and M. Sowwan, Sci. Rep. 3, 3083 (2013).

[25] H. Balamane, T. Halicioglu, and W. A. Tiller, Phys. Rev. B 46, 2250 (1992).

[26] J. F. Justo, M. Z. Bazant, E. Kaxiras, V. V. Bulatova, and S. Yip, MRS Symposium Proceedings, 469, 505 (1997).

[27] M. Z. Bazant, E. Kaxiras, and J. F. Justo, Phys. Rev. B 56, 8542 (1997).

[28] F. Mandl, Statistical Physics, 2nd ed. (Wiley, Chichester, UK, 1988).

[29] E. Kesälä, A. Kuronen, and K. Nordlund, Phys. Rev. B 75, 174121 (2007).
[30] R. C. Flagan and M. M. Lunden, Mater. Sci. Eng.: A 204, 113 (1995).

[31] T. Hihara and K. Sumiyama, J. Appl. Phys. 84, 5270 (1998).

[32] P. Erhart and K. Albe, Adv. Eng. Mater. 7, 937 (2005).

[33] A. Harjunmaa and K. Nordlund, Comput. Mater. Sci. 47, 456 (2009).

[34] P. V. Kashtanov, B. M. Smirnov, and R. Hippler, Physics Uspekhi 50, 455 (2007).

[35] Y.-H. Xu and J.-P. Wang, Adv. Mater. 20, 994 (2008).

[36] F. Wooten, K. Winer, and D. Weaire, Phys. Rev. Lett. 54, 1392 (1985).

[37] J. Samela, S. A. Norris, K. Nordlund, and M. J. Aziz, Nucl. Instr. Meth. Phys. Res. B 269, 1568 (2011).

[38] R. L. C. Vink, G. T. Barkema, M. A. Stijnman, and R. H. Bisseling, Phys. Rev. B 64, 245214 (2001).

[39] Y. Tu, J. Tersoff, G. Grinstein, and D. Vanderbilt, Phys. Rev. Lett. 81, 4899 (1998).

[40] K. Nordlund, M. Ghaly, R. S. Averback, M. Caturla, T. Diaz de la Rubia, and J. Tarus, Phys. Rev. B 57, 7556 (1998).

[41] K. Nordlund and R. S. Averback, Phys. Rev. B 59, 20 (1999).

[42] F. H. Stillinger and T. A. Weber, Phys. Rev. B 31, 5262 (1985).

[43] J. Samela, K. Nordlund, J. Keinonen, and V. N. Popok, Nucl. Instrum. Methods Phys. Res. B 255, 253 (2007).

[44] J. F. Ziegler, U. Littmark, and J. P. Biersack, The Stopping and Range of Ions in Solids (Pergamon, New York, 1985), p. 321.

[45] J. E. Jones, Proc. R. Soc. London, Ser. A 106, 463 (1924).

[46] K. Elihn, L. Landström, and P. Heszler, Appl. Surf. Sci. 186, 573 (2002).

[47] K. Nordlund and R. S. Averback, Phys. Rev. B 56, 2421 (1997).

[48] X. Yuan and A. Cormack, Comp. Mater. Sci. 24, 343 (2002).

[49] M. Hirasawa, T. Orii, and T. Seto, Appl. Phys. Lett. 88, 093119 (2006).

[50] P. Buffat and J.-P. Borel, Phys. Rev. A 13, 2287 (1976).

[51] L. J. Lewis, P. Jensen, and J.-L. Barrat, Phys. Rev. B 56, 2248 (1997).

[52] F. Baletto and R. Ferrando, Rev. Mod. Phys. 77, 371 (2005).

[53] J. Q. Broughton and X. P. Li, Phys. Rev. B 35, 9120 (1987).

[54] S. J. Cook and P. Clancy, Phys. Rev. B 47, 7686 (1993).

[55] J. Westergren, H. Grönbeck, S.-G. Kim, and D. Tománek, J. Chem. Phys. 107, 3071 (1997).

[56] K. C. Fang and C. I. Weng, Nanotechnol. 16, 250 (2005).

[57] H. M. Lu, P. Y. Li, Z. H. Cao, and X. K. Meng, J. Phys. Chem. C 113, 7598 (2009). 